\title{
A Multi-criteria Assessment of Deflection Methods for Dangerous NEOs
}

\author{
Joan Pau Sanchez Cuartielles, Camilla Colombo, \\ Massimiliano Vasile and Gianmarco Radice
}

Department of Aerospace Engineering, University of Glasgow, James Watt South Building, G128QQ, Glasgow, UK p.sanchez@aero.gla.ac.uk,c.colombo@aero.gla.ac.uk,m.vasile@aero.gla.ac.uk,g.radice@aero.gla.ac.uk

\begin{abstract}
Over the last few years, the possible scenario of an asteroid threatening to impact the Earth has stimulated an intense debate among the scientific community about possible deviation methods. In this paper we present a comparative assessment of some of the more feasible mitigation schemes reviewed in the literature (i.e. solar collector, nuclear blast, kinetic impactor, low-thrust propulsion and mass driver). For each strategy, a multi-criteria optimization method has been used to construct a set of Pareto optimal solutions, minimizing both the mass of the spacecraft and the warning time, while simultaneously maximizing the deviation. A dominance criterion has then been defined and used to compare all the Pareto sets. The achievable deviation at the MOID, either for a low-thrust or for an impulsive maneuver, has been computed through a set of analytical formulas. The variation in the orbit of the NEO has been estimated through a deviation action model that takes into account the wet mass of the spacecraft on the Earth. Finally the technology readiness level of each strategy has been used to recompute a more realistic value for the required warning time.
\end{abstract}

Keywords: Asteroids, Deviation, Mitigation, Solar Collector, Nuclear Blast

PACS: *91.62.Ty [Hazards, natural], 07.87.+v, 95.40.+s, 95.55.Pe [Artificial satellites], 96.30.Ys [Asteroids];

\section{INTRODUCTION}

Small celestial bodies like Near Earth Objects (NEOs) have become a common subject of study because of their importance to uncover the mysteries of the formation, evolution and composition of the solar system. Among all asteroids, NEOs have stepped into prominence because of two important aspects: they are among the easiest celestial bodies to reach from Earth, in some cases they can be reached with less demanding trajectories than a simple Earth-Moon trajectory and, even more meaningful, they may represent a threat to our planet.

On average, every $26-30$ million years a 10-km-sized asteroid strikes the Earth, while every 100 years there is a Tunguska class (100 $\mathrm{m}$ in size) asteroid impact. Each of these impacts permanently alters the characteristics of our planet to varying degrees. These events, and the risks for our fragile ecosystem, have made the space community turn their attention to the NEO issue [1]; several space mission to asteroids (e.g. Don Quijote [2], Deep Impact [3], NEAR [4] and ROSETTA [5]) have been designed in order to both improve the current knowledge of the solar system and to test the capability to mitigate a dangerous asteroid if the necessity should arise.

In order to predict the effects of a deflection strategy, some studies have addressed the asteroid deviation problem either with an analytical approach $[6,7,8]$ or by means of a numerical procedure based on a $n$-body model [9]. Previous deflection formulas were based on the modification of the orbital period due to a force acting on the asteroid. Only the effect on the orbital mean motion due to a change in the orbital energy was considered, and the variation in the other orbital elements was neglected. As a consequence the resulting deviation could be maximized only by applying some action in a direction parallel to the velocity vector of the asteroid. Any other strategy producing an action in any normal direction could not be investigated.

A more general approach was used by Park and Ross $[10,11]$ to determine the near-optimal direction of an impulsive maneuver. A similar approach was used by Conway [12]. 
During these last decades, several deviation methods involving numerous physical interactions with the asteroid have been analyzed. These strategies can be organized in four main groups: kinetic impactors, i.e. methods modeled as inelastic impacts [13,14]; attached propulsion devices [15] (e.g. electric/chemical engines, solar sails); induced thermo-optical changes on the asteroid surface [16] (e.g. induced Yarkovsky effect, paints) and ablation methods $[17,36]$ (e.g. lasers, solar mirrors).

A few authors have performed a partial comparative assessment of the numerous proposed mitigation strategies. Some of these emphasize a classification system based on NEO/spacecraft coupling [18], other systems are based on technology readiness and a third category on time to impact and/or intervention on the asteroid [19].

This paper presents a more exhaustive comparison of deflection methods according to different criteria. Three NEOs differing for physical characteristics (i.e. size, mass, shape and spin properties), composition (i.e. surface composition and heterogeneity, gas and dust emission) and orbital parameters (including inclination and eccentricity) have been selected for this analysis. Then, a group of mitigation strategies applied to these asteroids have been evaluated in terms of several figures of merit: achieved miss distance at the Earth, warning time, total mass into orbit and technology readiness considered here to be the estimated time to develop the required technology.

The warning time has been defined as the time difference between the impact epoch and the launch date that is required to achieve a given miss distance (or deviation), while the miss distance between the asteroid and the Earth is the displacement of the position of an asteroid at the Minimum Orbit Interception Distance (MOID) achieved by a deviation maneuver applied before the encounter. In order to study the effect of a general deflection strategy, we propose a set of analytical formulas that provides the variation of the MOID as a function of the variation of the orbital elements of the asteroid.

Finally the paper presents a multi-criteria optimization which provides a preliminary and relative measure of the effectiveness of one deviation over another.

\section{DEVIATION FORMULAS}

Given the time of interception $t_{d}$ for a generic NEO, the objective is to calculate the deviation achieved at the Minimum Orbit Interception Distance from the Earth, by applying a deviation action. The effect on the asteroid of all the deviation strategies analyzed has been modeled either as an impulsive $\delta \mathbf{v}$ at the interception time $t_{d}$ or as a continuous acceleration over an interval $\left[\begin{array}{ll}t_{d} & t_{e}\end{array}\right]$ where $t_{e}$ is the time when the thrusting arc ends. These two cases have been described by different models, based on the same approach: the miss distance achieved with a given deviation action has been computed by means of proximal motion equations [20] expressed as a function of the variation of the orbital elements. The variation of the orbital parameters has been computed with Gauss' planetary equations [21]. Proximal motion equations, already available in literature for formation flying with general elliptical orbits, provide, with minor modification, a simple and general means to compute, with good accuracy, the variation of the MOID.

Furthermore, the proposed analytical formulation can be used to compute the optimal direction of the deviating impulse and therefore the required $\delta \mathbf{v}$ to be imparted to the NEO for a given required variation of the MOID.

When the deviation action is modeled as a low-thrust action, Gauss' equations are numerically integrated to compute the variation of the orbital elements. The model developed has general validity and can be applied to every deviation strategy unless the deviated orbit is too distant from the original one.

\section{Impulsive Action Deviation Formulas}

The impulsive maneuver $\delta \mathbf{v}$ at time $t_{d}$ acts as a perturbation on the orbit of the NEO. The new orbit of the asteroid can be considered to be proximal to the unperturbed one (see Figure 1). 


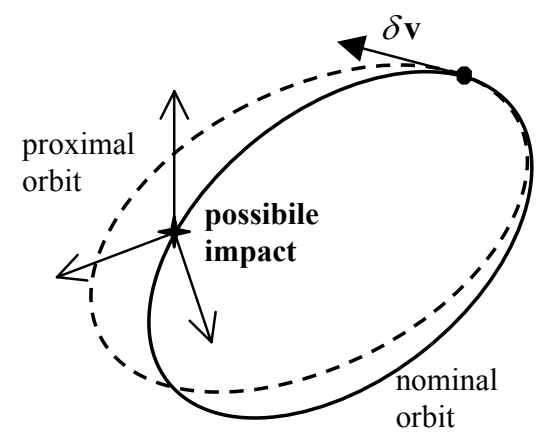

FIGURE 1. Chief orbit and proximal orbit.

If $\theta$ is the true anomaly of the NEO at the MOID along the unperturbed orbit and $\theta^{*}=\theta+\omega$ is the corresponding latitude, we can write the variation of the position of the NEO after deviation with respect to its unperturbed position by using the following proximal motion equations [20]:

$$
\begin{gathered}
\delta s_{r} \approx \frac{r}{a} \delta a+\frac{a e \sin \theta}{\eta} \delta M-a \cos \theta \delta e \\
\delta s_{\theta} \approx \frac{r}{\eta^{3}}(1+e \cos \theta)^{2} \delta M+r \delta \omega+\frac{r \sin \theta}{\eta^{2}}(2+e \cos \theta) \delta e+r \cos i \delta \Omega \\
\delta s_{h} \approx r\left(\sin \theta^{*} \delta i-\cos \theta^{*} \sin i \delta \Omega\right)
\end{gathered}
$$

where $\delta s_{r}, \delta s_{\theta}, \delta s_{h}$ are the displacements in the radial, transversal and perpendicular-to-the-orbit-plane directions respectively, so that $\delta \mathbf{r}=\left[\begin{array}{lll}\delta s_{r} & \delta s_{\vartheta} & \delta s_{h}\end{array}\right]^{T}$ and $\eta=\sqrt{1-e^{2}}$. The symbol $\delta$ is used to define the relative orbit in terms of the difference between the nominal orbit and the perturbed one. The variation of the orbital parameters $a, e$, $i, \omega$ and $\Omega$ are computed through Gauss' planetary equations [21] considering an instantaneous change in the NEO velocity vector $\delta \mathbf{v}=\left[\begin{array}{lll}\delta v_{t} & \delta v_{n} & \delta v_{h}\end{array}\right]^{T}:$

$$
\begin{aligned}
& \delta a=\frac{2 a^{2} v}{\mu} \delta v_{t} \\
& \delta e=\frac{1}{v}\left[2(e+\cos \theta) \delta v_{t}-\frac{r}{a} \sin \theta \delta v_{n}\right] \\
& \delta i=\frac{r \cos \theta^{*}}{h} \delta v_{h} \\
& \delta \Omega=\frac{r \sin \theta^{*}}{h \sin i} \delta v_{h} \\
& \delta \omega=\frac{1}{e v}\left[2 \sin \theta \delta v_{t}+\left(2 e+\frac{r}{a} \cos \theta\right) \delta v_{n}\right]-\frac{r \sin \theta^{*} \cos i}{h \sin i} \delta v_{h} \\
& \delta M_{t_{d}}=-\frac{b}{e a v}\left[2\left(1+\frac{e^{2} r}{p}\right) \sin \theta \delta v_{t}+\frac{r}{a} \cos \theta \delta v_{n}\right]
\end{aligned}
$$

The Gauss' equations have been written in a tangential-normal-binormal reference frame, so that $\delta v_{t}$ and $\delta v_{n}$ are the components of the impulsive deviation maneuver, in the plane of the osculation orbit, along the velocity vector and perpendicular to it. 
The variation $\delta M_{t_{d}}$ takes into account only the instantaneous change of the orbit geometry at time $t_{d}$. On the other hand, due to the change in the semi-major axis, there is a variation of the mean motion $n$ with the associated variation in the mean anomaly:

$$
\delta M_{n}=\delta n\left(t_{M O I D}-t_{d}\right)=\delta n \Delta t
$$

where $t_{\text {MOID }}$ is the time at the MOID along the orbit of the NEO and $\delta n=\sqrt{\frac{\mu}{a^{3}}}-\sqrt{\frac{\mu}{(a+\delta a)^{3}}}$. Eq. (5) takes into account the phase shifting between the Earth and the NEO. The total variation in the mean anomaly between the unperturbed and the proximal orbit is therefore:

$$
\delta M=-\frac{b}{e a v}\left[2\left(1+\frac{e^{2} r}{p}\right) \sin \theta \delta v_{t}+\frac{r}{a} \cos \theta \delta v_{n}\right]+\delta n \Delta t
$$

The result in Eq. (6) can be proved as follows. Let us define $\tilde{M}_{M O I D}$ as the mean anomaly of the perturbed orbit at the MOID and $M_{\text {MOID }}$ the mean anomaly of the nominal orbit at the MOID:

$$
\begin{aligned}
& \tilde{M}_{M O I D}=M\left(t_{d}\right)+(n+\delta n)\left(t_{M O I D}-t_{d}\right)+\delta M_{t_{d}}=n\left(t_{d}-t_{0}\right)+(n+\delta n)\left(t_{M O I D}-t_{d}\right)+\delta M_{t_{d}} \\
& M_{M O I D}=n\left(t_{M O I D}-t_{0}\right)
\end{aligned}
$$

Then,

$$
\delta M=\tilde{M}_{M O I D}-M_{M O I D}=\delta n\left(t_{M O I D}-t_{d}\right)+\delta M_{t_{d}}
$$

Note that Eqs. (1) to (3) are an approximation of the first order and thus can be used only if the relative orbit radius $\delta \mathbf{r}$ is small compared to the nominal orbit radius $\mathbf{r}$.

The accuracy of the deviation formulas has been assessed by numerically propagating forward in time the deviated orbit of the asteroid and comparing the obtained variation in the position vector with the one predicted by Eqs. (1) to (4). The nominal trajectory has been propagated from the deviation point up to the MOID, in the interval $\left[\begin{array}{ll}t_{d} & t_{M O I D}\end{array}\right]$ and the deviated trajectory has been integrated starting from the deviation point on the NEO orbit with the modified velocity vector $\mathbf{v}+\delta \mathbf{v}$. As a measure of accuracy we used the relative error between the variation in position computed numerically and analytically:

$$
e_{r}=\frac{\left\|\delta \mathbf{r}_{\text {propagated }}-\delta \mathbf{r}_{\text {estimated }}\right\|}{\left\|\delta \mathbf{r}_{\text {propagated }}\right\|}
$$

Figures 2 and 3 show the relative errors as a function of the anticipating time $t_{M O I D}-t_{d}$ and $\delta \mathbf{v}_{t}$ along track (i.e. along the instantaneous velocity vector of the NEO). The relative error has been calculated for the asteroid SG344 and for the asteroid Quetzalcoatl. These two asteroids with widely different orbits, the former with $e<0.1$ and $i<10^{\circ}$, the latter with $e>0.1$ and $i>10^{\circ}$, have been chosen in order to study the influence of the orbital parameters on the accuracy of the deflection formulas.

Now if $\Delta \mathbf{r}=\left[\begin{array}{llll}\Delta s_{r} & \Delta s_{\theta} & \Delta s_{h}\end{array}\right]^{T}$ is the vector distance of the asteroid from the Earth at the MOID, and $\delta \mathbf{r}=\left[\begin{array}{lll}\delta s_{r} & \delta s_{\theta} & \delta s_{h}\end{array}\right]^{T}$ is the variation given by Eqs. (1) to (3), then the objective function for the maximum deviation problem is the following:

$$
J=\left(\Delta s_{r}+\delta s_{r}\right)^{2}+\left(\Delta s_{\theta}+\delta s_{\theta}\right)^{2}+\left(\Delta s_{h}+\delta s_{h}\right)^{2}
$$

Note that the objective function in Eq. (9) intrinsically requires causing a variation that increases the MOID. 

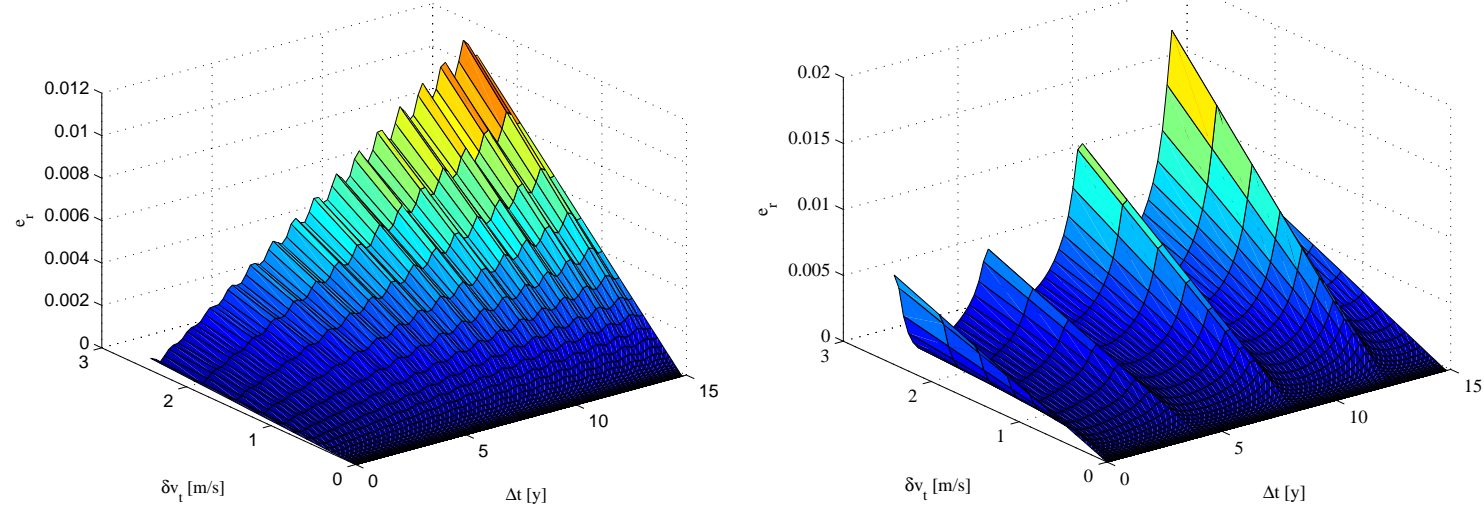

FIGURE 2. Relative error for SG344 deviation.

FIGURE 3.

Relative error for Quetzalcoatl deviation.

\section{Optimal Action Directions Analysis}

Prior to the multi-criteria optimization, an analysis has been performed in order to find the optimal direction of the impulsive maneuver to be imparted to the asteroid, at a specific time, so to achieve the maximum deviation at the MOID. If Eq. (6) is incorporated into the system in Eq. (4) and along with Eqs. (1) to (3), written in matrix form, then we can compute the transition matrix that links $\delta \mathbf{v}$ at $t_{d}$ to $\delta \mathbf{r}$ at $t_{M O I D}$ :

$$
\left\{\begin{array}{l}
\delta \mathbf{r}\left(t_{M O I D}\right)=\mathbf{A}_{M O I D} \delta \boldsymbol{\alpha}\left(t_{d}\right) \\
\delta \boldsymbol{\alpha}\left(t_{d}\right)=\mathbf{G}_{d} \delta \mathbf{v}\left(t_{d}\right)
\end{array} \Rightarrow \delta \mathbf{r}\left(t_{M O I D}\right)=\mathbf{A}_{M O I D} \mathbf{G}_{d} \delta \mathbf{v}\left(t_{d}\right)=\mathbf{T} \delta \mathbf{v}\left(t_{d}\right)\right.
$$

where $\delta \boldsymbol{\alpha}\left(t_{d}\right)=\left[\begin{array}{llllll}\delta a & \delta e & \delta i & \delta \Omega & \delta \omega & \delta M\end{array}\right]^{T}$ is the orbital element difference, $\mathbf{A}_{M O I D}$ and $\mathbf{G}_{d}$ are the matrices associated respectively to Eqs. (1) to (4). The subscript indices, MOID and $d$, indicate that the matrices are calculated respectively at $t_{M O I D}$ and $t_{d}$.

Following Conway's approach [12], we maximize $\left\|\delta \mathbf{r}\left(t_{\text {MOID }}\right)\right\|=\max \left(\mathbf{T} \delta \mathbf{v}\left(t_{d}\right)\right)$ by choosing an impulse vector $\delta \mathbf{v}\left(t_{d}\right)$ parallel to the eigenvector $\mathbf{n}$ of $\mathbf{T}^{T} \mathbf{T}$ conjugated to the maximum eigenvalue. Figure 4 emphasizes the optimality of the solution: the deviation obtained with $\|\delta \mathbf{v}\|=0.07 \mathrm{~m} / \mathrm{s}$ has been calculated, applying the maneuver along the optimal direction (solid line), and along the motion direction (dot line), the normal to the motion (dashdotted line) and the $h$-direction (dashed line), as a function of the anticipation time $\Delta t$ expressed as a multiple of the NEO orbital period. $\|\delta \mathbf{r}\|$ associated to $\delta \mathbf{v}_{\text {opt }}$ is the maximum one and overlaps the deviation achieved with a normal impulse, for low $\Delta t$, and the one with a tangent maneuver, for bigger $\Delta t$.

As a result of this analysis we can derive the following conclusions (see Fig. 4): for a specific $\Delta t_{N E O}$, different for every asteroid, the normal component dominates the other two, while for larger $\Delta t$, the tangential component becomes dominant. Moreover, at exact multiples of $T_{N E O}$ prior to $t_{M O I D}$ the normal component goes to zero because it does not affect the deviation. Finally the out-of-plane component $h$ of $\delta \hat{\mathbf{v}}_{\text {opt }}$ is negligible compared to the other two for large $\Delta t$ but becomes comparable for short anticipating times. These conclusions are consistent with the results presented by Park and Ross [10] and by Conway [12]. 


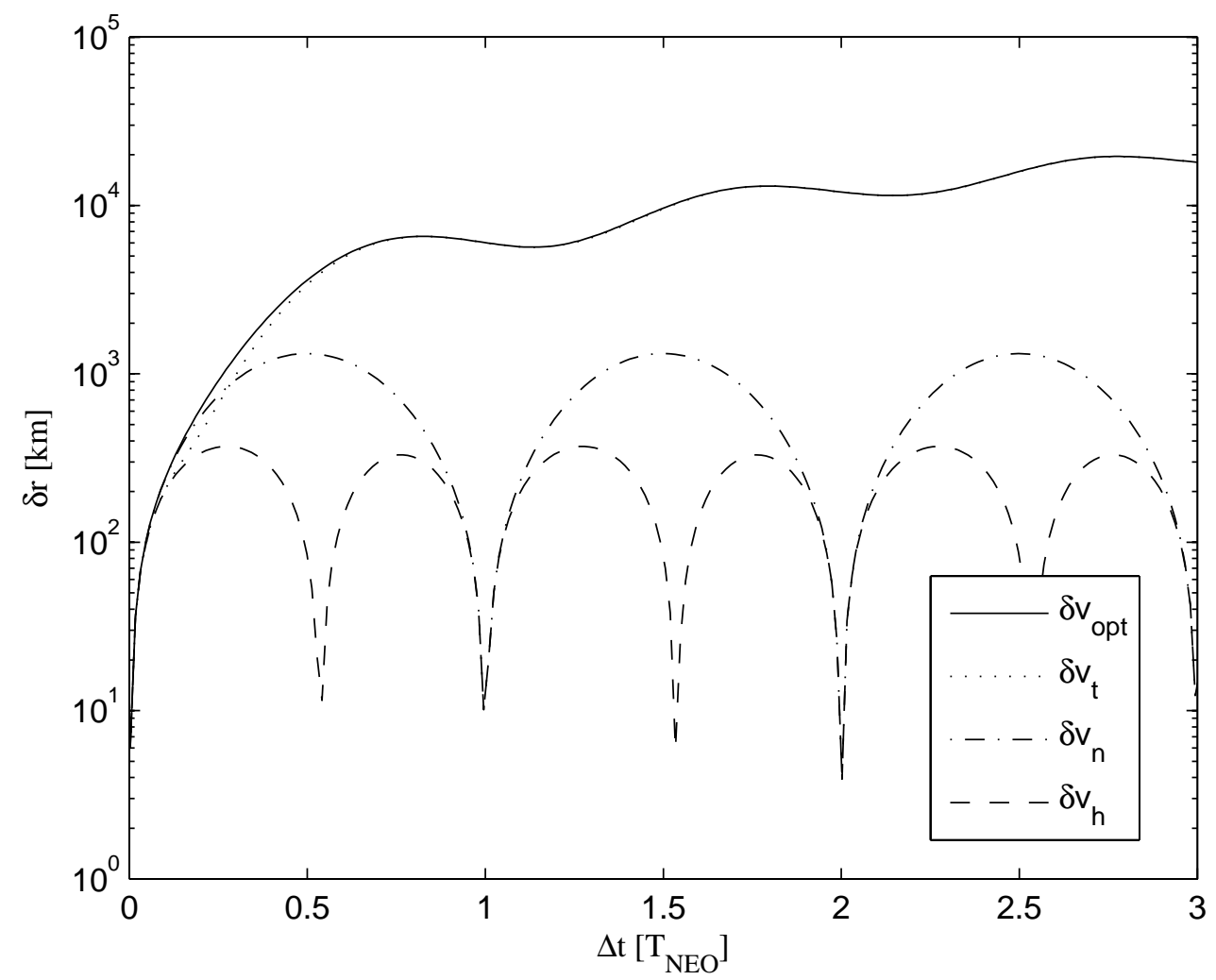

FIGURE 4. Deviation achieved with $\|\delta v\|=0.07 \mathrm{~m} / \mathrm{s}$ for the asteroid 2000SG344.

\section{Low-Thrust Action Deviation Formulas}

When a continuous deviation action is applied, the total variation in the orbital parameters is computed by numerically integrating Gauss' variational equations over the interval $\left[\begin{array}{ll}t_{d} & t_{e}\end{array}\right]$ :

$$
\begin{aligned}
& \frac{d a}{d t}=\frac{2 a^{2} v}{\mu} a_{t} \\
& \frac{d e}{d t}=\frac{1}{v}\left[2(e+\cos \theta) a_{t}-\frac{r}{a} \sin \theta a_{n}\right] \\
& \frac{d i}{d t}=\frac{r \cos \theta^{*}}{h} a_{h} \\
& \frac{d \Omega}{d t}=\frac{r \sin \theta^{*}}{h \sin i} a_{h} \\
& \frac{d \omega}{d t}=\frac{1}{e v}\left[2 \sin \theta a_{t}+\left(2 e+\frac{r}{a} \cos \theta\right) a_{n}\right]-\frac{r \sin \theta^{*} \cos i}{h \sin i} a_{h} \\
& \frac{d M}{d t}=n-\frac{b}{e a v}\left[2\left(1+\frac{e^{2} r}{p}\right) \sin \theta a_{t}+\frac{r}{a} \cos \theta a_{n}\right]
\end{aligned}
$$


where $\mathbf{a}=\left[\begin{array}{lll}a_{t} & a_{n} & a_{h}\end{array}\right]^{T}$ is the acceleration vector, given by the continuous thrust. Note that in this case the derivative of $M$, also takes into account the change in mean anomaly due to the change in the angular velocity $n$ on the thrusting arc, because the term $n$ cannot be ignored as is done for the impulsive maneuver case. If $\mathbf{a}(t)=\left[\begin{array}{llllll}a & e & i & \Omega & \omega & M\end{array}\right]^{T}$ is the vector of the orbital parameters, their variation in the interval $\left[\begin{array}{ll}t_{d} & t_{e}\end{array}\right]$ is:

$$
\Delta \mathbf{a}=\mathbf{a}\left(t_{d}\right)-\mathbf{a}\left(t_{e}\right)=\left[\begin{array}{llllll}
\Delta a & \Delta e & \Delta i & \Delta \Omega & \Delta \omega & \Delta M
\end{array}\right]^{T} .
$$

We can note that $\delta a=\Delta a, \delta e=\Delta e, \delta i=\Delta i, \delta \Omega=\Delta \Omega, \delta \omega=\Delta \omega$ can be substituted in the proximal motion equations, while $\delta M$ has to be computed in a way analogous to (6). Since:

$$
\begin{aligned}
& \tilde{M}_{M O I D}=M\left(t_{e}\right)+n_{e}\left(t_{M O I D}-t_{e}\right)=M\left(t_{d}\right)+\Delta M+n_{e}\left(t_{M O I D}-t_{e}\right)=n\left(t_{d}-t_{0}\right)+\Delta M+n_{e}\left(t_{\text {MOID }}-t_{e}\right) \\
& M_{\text {MOID }}=n\left(t_{\text {MOID }}-t_{0}\right)
\end{aligned}
$$

we can derive $\delta M$ as:

$$
\delta M=\tilde{M}_{M O I D}-M_{M O I D}=\left(n_{e}-n\right) t_{M O I D}+n t_{d}-n_{e} t_{e}+\Delta M
$$

where $n$ is the nominal angular velocity and $n_{e}-n=\Delta n$. At this point Eqs. (1) to (3) can be used to compute the resulting miss distance $\delta \mathbf{r}$.

\section{ACTION MODELS}

In order to evaluate the performance achievable with each deviation strategy, a set of simple models has been developed. Each model yields the total impulse imparted to the asteroid and the total required spacecraft mass. The general analytical form of all the models is:

$$
\begin{aligned}
& \Delta \mathbf{v}=f\left(m_{d}, I d_{N E O}\right) \\
& \text { Thrust }=f\left(m_{d}, I d_{N E O}\right)
\end{aligned}
$$

where $m_{d}$ is dry mass of the spacecraft, which is defined as the mass available to alter the trajectory of the celestial body after the transfer from Earth to the asteroid, and $I d_{N E O}$ is the asteroid identification number.

\section{Solar Collector}

For this study, the solar collector is assumed to be an inflatable mirror that focuses enough energy onto the asteroid surface to ablate it. The evaporated material produces a plume of gas, which provides a constant thrust. A comprehensive explanation of the model can be found in [22], with a similar model in [23].

\section{Electric Propulsion}

In this case the linear momentum of the asteroid is perturbed by a thrust given by a propulsion system attached to the surface of the asteroid.

\section{Spacecraft Sizing}

The system consists of a minimum of two engines situated at opposite spots along the equator of the asteroid. By properly scheduling the periods when the engines are switched on and off, we can obtain a constant thrust and a limited scattering factor. The scattering factor takes into account the misalignment from the optimal thrusting 
direction. Assuming that the power available comes from a subsystem, the weight of which is $50 \%$ of the dry mass, and that the power obtainable is about $20 \mathrm{~W} / \mathrm{kg}$ [24], the thrust can be calculated using an averaged thrust/power ratio for the most common Ion thrusters [24] of $34 \mathrm{mN} / \mathrm{kW}$. This hypothesis leads to the following relation:

$$
\text { Thrust }=s_{\text {Scattering }} \cdot 0.5 \cdot \frac{m_{d}}{2} \cdot \frac{1}{0.02[\mathrm{~kW} / \mathrm{kg}]} \cdot 0.034[\mathrm{~N} / \mathrm{kW}]
$$

Finally, the mass consumption or propellant mass is calculated using the rocket equation with an $I_{s p}$ of $3080 \mathrm{~s}$.

\section{Mass Driver}

The idea behind this strategy is to change the linear momentum of the asteroid by using some ejected asteroid material. This ejected material would be accelerated by a catapult or a gun system, able to convert the available spacecraft power into kinetic energy.

$$
K E=\frac{1}{2} m \cdot v_{e}^{2}
$$

Obviously the conversion of energy from electric to kinetic is far from optimal, but we could assume that between the power system and payload (e.g. catapult or gun) the spacecraft is able to convert $50 \%$ of the available energy into kinetic energy [25] (i.e. 50\% of mechanical loss). The excess velocity of the expelled material and the mass expelled with each shot are two parameters that would come from the engineering design of the system. In literature a value of 100-300 km/s has been identified as a realistic excess velocity from which expelled mass can be estimated $[25,26]$.

Once the spacecraft has landed and the operations have started, it would dig and collect material during most of its operating time and shoot this material when pointing within 5 degrees of the orbital tangential direction.

\section{Spacecraft Sizing}

In our case $50 \%$ of the dry mass has been considered to be power system. We average the specific power of several power systems, since the scope of this paper is to compare deviation methods and not to discuss the best engineering design. The ratio obtained is $20 \mathrm{~W} / \mathrm{kg}$ [24] and therefore the power available is known. With the rotation period and the configuration described above, it is straightforward to calculate the time available to shoot. Consequently the energy is known, and since we have assumed an excess velocity of $200 \mathrm{~km} / \mathrm{s}$ the expelled mass determined using Eq. (11).

$$
\begin{aligned}
m_{\text {payload }} & =(50 \%) \cdot m_{d} \\
\text { Power } & =20[\mathrm{~W} / \mathrm{kg}] \cdot m_{\text {payload }} \\
m_{\text {launch }} & =\frac{(50 \%) \cdot 2 \cdot \text { Power } \cdot \text { Period }_{\text {rotation }} \cdot\left(\frac{10^{\circ}}{360^{\circ}}\right)}{V_{e}^{2}}
\end{aligned}
$$

The change in the velocity of the asteroid is determined using the conservation of linear momentum and taking into account the change in the asteroid mass due to every shot.

$$
\delta \mathbf{v}=\frac{m_{\text {launch }}}{m_{N E O}(t)} v_{e}
$$

At each $\delta \mathbf{v}$ there is a corresponding finite variation in orbital elements and the new set of orbital parameters must be calculated before the subsequent impulsive action. The finite change in the true anomaly has been added to the Gauss' equations (4) such that: 


$$
\delta \theta_{t_{d}}=-\frac{1}{e v}\left[2 \sin \theta \delta v_{t}+\left(2 e+\frac{r}{a} \cos \theta\right) \delta v_{n}\right]
$$

The mean anomaly before each mass ejection is computed from the first equation in (7) and then Kepler's equation is solved for the osculating eccentric anomaly and the true anomaly, by the Newton method described in [21].

\section{Nuclear Blast}

This scheme is based on the use of nuclear warheads as the energy source. The interaction between the nuclear blast and asteroid has been modeled by means of neutron radiation, X-rays and debris from the explosion.

\section{System Definition \& Architecture}

The asteroid is modeled as a spherical body with a known density and mass; accordingly the asteroid radius comes from a straightforward calculation. The composition of the asteroid has been averaged from ordinary and carbonaceous chondrites (i.e. meteoroids), therefore its physical properties have been averaged as well.

The spacecraft carries $30 \%$ of its mass in payload. This payload will be a fusion device in order to maximize the neutron radiation, which, as will be shown later, achieves the best results. The energy available from the explosion is calculated using the ratio $0.75 \mathrm{ktons} / \mathrm{kg}[27,28]$

$$
\begin{aligned}
& \text { Nuclear }_{\text {head }}=(30 \%) \cdot m_{d} \\
& \text { Energy }[\mathrm{ktons}]=0.75\left[\mathrm{ktons} \cdot \mathrm{kg}^{-1}\right] \cdot \text { Nuclear }_{\text {head }}
\end{aligned}
$$

The spacecraft payload will be detonated at a certain distance from the asteroid. This distance $(H)$, shown in Figure 5, is a very important parameter in the model, having a direct relation with the efficiency of the energy coupling. This altitude of detonation was fixed at $H=0.077 R_{a}$, which was shown to maximize the energy coupling.

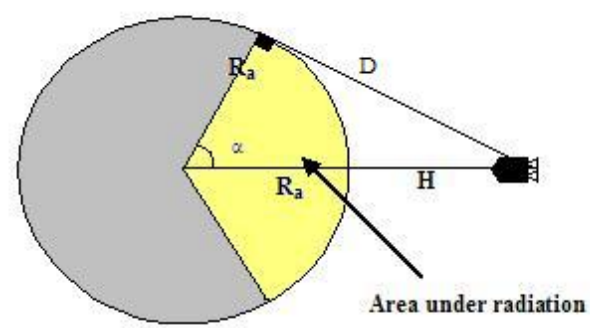

FIGURE 5. Nuclear Blast model outline.

Model

The energy distribution of a fusion device used here agrees with [29]; $95 \%$ of the energy is shared by neutron radiation (20\%), X-rays (55\%) and kinetic energy in debris $(20 \%)$.

For the debris' case, the velocity of the spacecraft mass is calculated using the kinetic energy equation. In order to compute the total amount of debris that impacts the asteroid, the ratio $S$ between the total area of the shock wave and the area that intersects the asteroid is determined.

$$
\begin{aligned}
& V_{\text {debris }}=\sqrt{\frac{2 \cdot 0.2 \cdot \text { Energy }}{m_{d}}} \\
& S=\frac{1}{2}-\frac{\sqrt{H}}{2} \cdot \frac{\sqrt{H+2 R_{a}}}{R_{a}+H} \\
& m_{\text {debris }}=m_{d} \cdot S
\end{aligned}
$$


The final increment in the asteroid velocity is calculated using linear momentum conservation, applying a scattering factor and a certain momentum enhancement [30].

$$
\Delta V_{\text {debris }} \approx B_{\text {enhance }} \cdot S_{\text {Scattering }} \cdot \frac{m_{\text {debris }} \cdot V_{\text {debris }}}{M_{\text {asteroid }}}
$$

For the radiation case the energy absorbed per unit of mass is $E_{\text {absorved }}=\mu \cdot E$, where $\mu\left[\mathrm{m}^{2} / \mathrm{kg}\right]$ is the opacity of a material to a certain radiation (i.e. neutron or X-rays) and $E$ is the energy per unit of area. Therefore the penetration $x$ of this radiation inside the asteroid will be:

$$
E(x)=E_{0} e^{-\rho \mu x}
$$

Where $E_{0}$ is the energy at the surface that obviously depends on the distance from the explosion and $\rho$ is the density of the asteroid. Then, from the total energy absorbed by a certain mass we subtract the sublimation energy $E_{v}$, which in this model has been set to $5.03 \mathrm{~kJ} / \mathrm{g}$ [31]. The velocity of the expelled mass is then easily calculated using $K E=0.5 \cdot m V^{2}$. Consequently, the linear momentum per unit area gained by the asteroid due to the sublimated mass is:

$$
\begin{gathered}
d p \approx \rho \cdot \sqrt{2 \cdot\left(E(x)-E_{v}\right)} \cdot d x \\
P=\sqrt{2} \cdot \rho \cdot \int_{0}^{X_{\max }}\left(E_{0}(h) e^{-\rho \mu x}-E_{v}\right)^{1 / 2} d x
\end{gathered}
$$

where $X_{\max }$ is the maximum depth where the evaporation takes place, which is calculated by:

$$
X_{\max }=\frac{1}{\rho \mu} \ln \left(\frac{\mu E_{0}}{E_{v}}\right)
$$

We now have to integrate over the entire radiated surface. Since every point on the surface has a different distance to the asteroid, we have a double integration:

$$
P=2 \pi r \cdot \sqrt{2} \rho \int_{H}^{D} \int_{0}^{X_{\max }}\left(\frac{\text { Ratio } \cdot \text { Energy }}{4 \pi h^{2}} e^{-\rho \mu x}-E_{v}\right)^{1 / 2} d x \cdot d h
$$

Where Ratio is the fraction of energy corresponding to a certain radiation (i.e. neutron or X-rays), Energy is the total energy of the nuclear device in Joules, and $h$ is the distance from the surface to the explosion, which goes from a minimum distance $H$, the altitude of the spacecraft from the asteroid surface, to a maximum distance $D$, which is the distance to the horizon of the asteroid as viewed by the spacecraft. Finally, the $\Delta \mathbf{v}_{\text {radiation }}$ experienced by the asteroid is calculated by integrating Eq. (14) and dividing it by the total mass of the asteroid.

Since the physical properties of the asteroid were averaged from the physical properties of chondrites, the opacities or absorption coefficients for neutron radiation and X-rays have been chosen to be $\mu_{\text {neutron }}=0.0044 \mathrm{~m}^{2} / \mathrm{kg}$ and $\mu_{X-r a y}=5 \mathrm{~m}^{2} / \mathrm{kg}$ respectively.

After the initial results it became obvious that the nuclear radiation provides the best contribution to the overall performance of the nuclear strategy. Although X-rays give very high excess velocities, neutron radiation produces more evaporation of mass, therefore being more efficient in providing an impulse to the asteroid. For this reason, as they maximize the neutron radiation, fusion devices have been adopted in this model. To conclude, the total impulsive action is: 


$$
\Delta \mathbf{v}=\Delta \mathbf{v}_{\text {radiation }}+\Delta \mathbf{v}_{X \text {-rays }}+\Delta \mathbf{v}_{\text {debris }}+\Delta \mathbf{v}_{\text {neutrons }}
$$

\section{Kinetic Impactor}

The Kinetic Impactor is the simplest concept of asteroid hazard mitigation. Here the asteroid linear momentum is modified by the collision of a certain mass, generally the spacecraft. This collision is modeled as a simple inelastic impact with a certain momentum enhancement [13].

This factor takes into account that, despite the momentum provided to the initial mass of the asteroid is calculated only using the inelastic collision model, the momentum of the asteroid itself is enhanced by the blast of material expelled after the collision. In order to be conservative in our models, the value of the enhancement chosen is 2 [13]. The variation of velocity imparted by the spacecraft (i.e. impactor) is therefore given by:

$$
\delta \mathbf{v}=\beta \frac{m_{S / C}}{\left(m_{N E O}+m_{S / C}\right)} \Delta \mathbf{v}_{S / C}
$$

Where $\beta$ is the momentum enhancement factor and the relative velocity of the spacecraft with respect to the asteroid at the deviation point $\Delta \mathbf{v}_{S / C}$ is computed from the solution of a Lambert's problem. Consequently the direction of the $\delta \mathbf{v}$ provided depends on the arrival and launch dates.

\section{TRANSFER TRAJECTORY}

The transfer path and the consequent propellant mass required have been determined with two techniques, depending on the propulsion system used. For high-impulse systems, such as the Kinetic Impactor and the Nuclear Blast, a two-impulse Lambert's solver was used, with a specific impulse of $315 \mathrm{~s}$ for propellant calculation, while for low-thrust trajectories a shape-based approach was adopted instead [32], with a specific impulse of $2500 \mathrm{~s}$.

\section{OPTIMIZATION PROCEDURE}

The optimality of each strategy is here defined through a number of criteria or objectives that have to be attained. Unlike single objective problems, multiple objective problems look for a set of optimal values rather than a single optimal one. The general problem is to find a set $X$ of feasible solutions $\mathbf{x}$ such that the property $P(\mathbf{x})$ is true for all $\mathbf{x} \in X \subseteq D:$

$$
X=\{\mathbf{x} \in D \mid P(\mathbf{x})\}
$$

where the domain $D$ is a hyperrectangle defined by the upper and lower bounds on the components of the vector $\mathbf{x}$ :

$$
D=\left\{x_{i} \mid x_{i} \in\left[b_{i}^{l}, b_{i}^{u}\right] \subseteq \mathfrak{R}, i=1, \ldots, n\right\}
$$

All the solutions satisfying property $P$ are here defined to be optimal with respect to $P$ or $P$-optimal and $X$ can be said to be a $P$-optimal set. In the case of multiobjective optimization, if $P$ is a dominance condition or Pareto optimality condition for the solution $\mathbf{x}$, then the solution is Pareto-optimal if $P(\mathbf{x})$ is true. According to the definition, a set of parameters is Pareto optimal if there exists no other feasible vector of variables which would decrease some criterion without causing a simultaneous increase in at least one other criterion.

The search of the P-optimal sets $X$, for each strategy, is here reconstructed through a hybridization of a domain decomposition technique combined with an agent-based search approach [33]. Each function vector $j$ is associated to a scalar dominance index $I_{d}$ such that:

$$
I_{d}\left(\mathbf{x}_{j}\right)=\left|\left\{i \mid i \in N_{p} \wedge \mathbf{x}_{i} \succ \mathbf{x}_{j}\right\}\right|
$$


where the symbol || is used to denote the cardinality of a set, $\succ$ represents the dominance of the $\mathbf{x}_{i}$ solution over the $\mathbf{x}_{\mathbf{j}}$ solution and $N_{p}$ is the set of the indices of all the agents in the population. The property $P(\mathbf{x})$ in this case simply defines non-dominated solutions:

$$
X=\left\{\mathbf{x} \in D \mid I_{d}(\mathbf{x})=0\right\}
$$

\section{Objective Function Definition}

Three figures of merit have been selected to define the optimality of each strategy:

- the warning time $t_{w}=t_{l}-t_{M O I D}$, which is the interval between the launch date and the time at the MOID;

- the mass in space $m_{0}$ is the wet mass at the Earth, after launch;

- the total deviation $\Delta \mathbf{r}$ at the MOID.

The $t_{M O I D}$ is the first date at which the asteroid reaches the minimum interception distance from the Earth orbit in a given range of times. Note that the Earth is not necessarily at that physical point in the orbit; the aim of this analysis is to measure the achieved deviation and not to reproduce a real impact scenario. In order for the hypotheses of the proximal motion equations to hold true, the relative orbit radius $\delta \mathbf{r}$ must be small compared with the nominal orbit radius $\mathbf{r}$. Therefore the maximum acceptable deviation has been set to the Earth-Moon distance, since this is considered to be sufficient to ward off the threat of an impact.

\section{RESULTS}

Since the effectiveness of a given strategy can depend on the physical characteristics of the NEO it is applied to, three asteroids (see Table 1) belonging to the three NEO categories (Apollo, Atens and Amor) have been chosen for the multicriteria analysis. These asteroids differ for their mass, composition and orbital elements.

TABLE 1. Asteroids selected for analysis.

\begin{tabular}{ll}
\hline NEO Category & Designation \\
\hline Atens & Apophis \\
Apollo & Castalia \\
Amor & Nyx \\
\hline
\end{tabular}

The result of each deflection strategy for each of these asteroids is represented by a set of Pareto fronts. As an example, we present here all the solutions for Apophis (Figure 6 - Figure 10). In order to improve the visualization of the Pareto fronts, for each figure an approximating surface has been generated from the scattered set of Pareto optimal solutions.

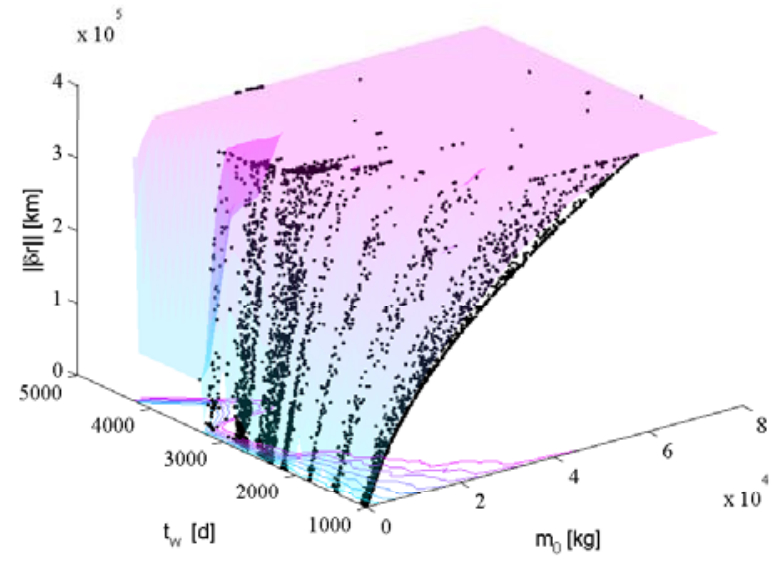

FIGURE 6. Nuclear blast Pareto front for Apophis.

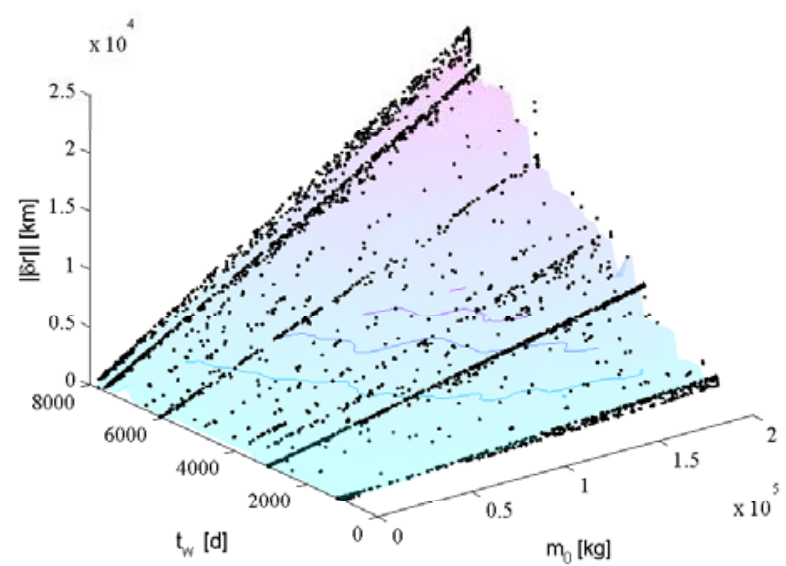

FIGURE 7. Kinetic Impactor Pareto front for Apophis. 
The two methods that exhibit the best results for almost all the asteroids analyzed are the Solar and the Nuclear strategies (see Figs. 8 and 6); in fact they reach the limit that was set on the maximum deviation for small values of the wet mass and the warning time. Notice that the two approaches are entirely different as the deviation is achieved through a continuous thrusting arc in the former case or through an impulsive change in the linear momentum in the latter case.

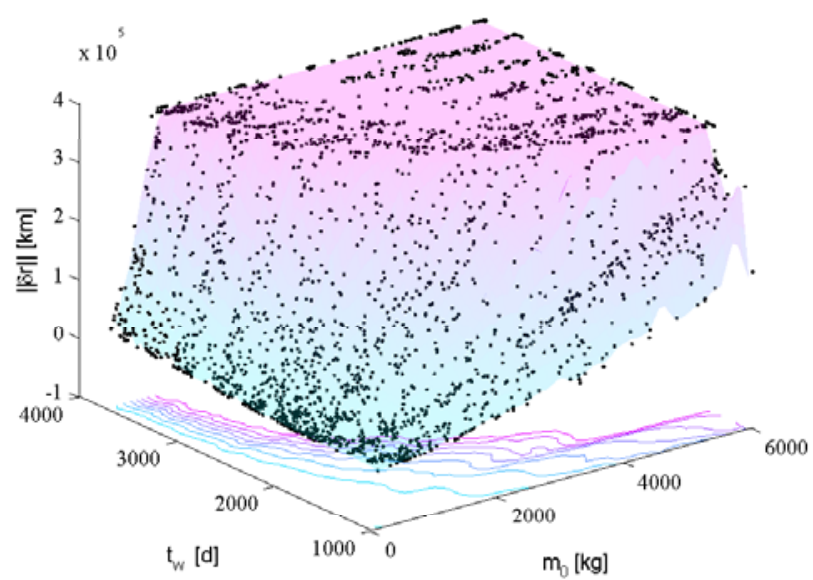

FIGURE 8. Solar Collector Pareto front for Apophis.

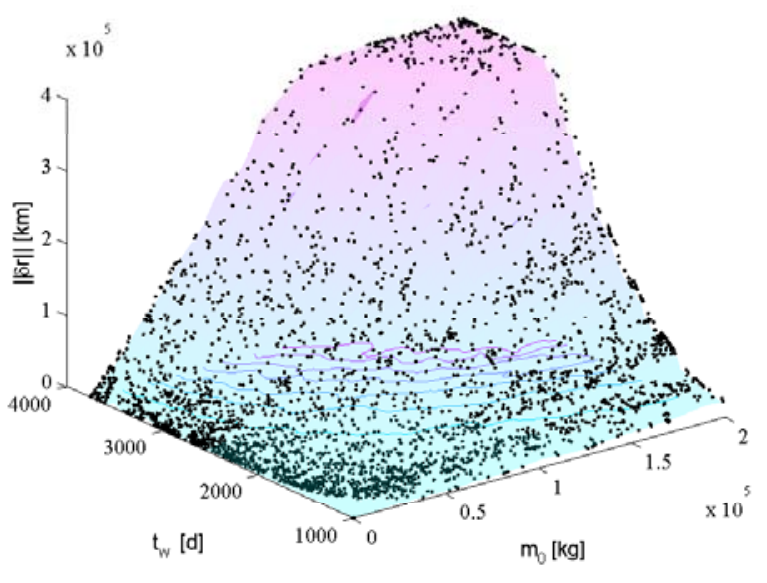

FIGURE 9. Mass Driver Pareto front for Apophis.

Mass Driver (Fig. 9) shows a satisfactory performance, reaching the maximum deviation in some cases. LowThrust (Fig. 10) and Mass Driver (Fig. 9) have a similar behavior because, even if the latter has been modeled as a multi-impulsive-action, its effect is analogous to a continuous thrust. From this initial investigation, the Kinetic Impactor, shown in Fig. 7 does not seem to be competitive compared to the other strategies.

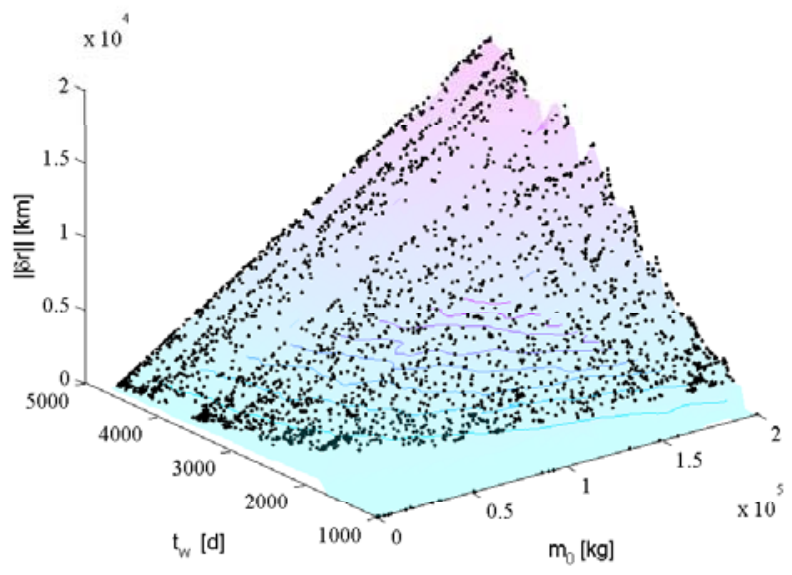

FIGURE 10. Low-Thrust Pareto front for Apophis.

Despite the physical and orbital differences among the NEO's in Table 1, the shape of the Pareto fronts is mostly dependent on the mitigation strategy used. NEO orbital characteristics, size and rotational period play an important role in modeling the surface of the Pareto front, sizing it and changing slightly the inclination and position in the criteria space. All the computed Pareto fronts for all the asteroids have a number of common features. In particular, the linear or quadratic increase of the deviation with $m_{0}$ (initial mass), which is directly related to the analytic models presented in this paper, and the periodicity along the warning time $\left(t_{w}\right)$ axis which is directly related to the point along the orbit where a variation of the asteroid velocity is most effective. Note that for low thrust propulsion, this periodicity appears only for short warning times. This is consistent with the fact that for long warning times the choice of an optimal point along the orbit is less crucial. 


\section{Multi-Criteria Analysis}

The effectiveness and efficiency of each strategy are expressed through a set of Pareto optimal points. In order to compare one strategy against the others it is necessary to define the concept of dominance of one Pareto set over another. An element (or solution belonging to the Pareto set) $i$ of strategy $A$ is said to dominate an element $j$ of strategy $B$ if all the components of the vectorial objective function $\mathbf{f}_{j}^{B}$ are smaller than all the components of the vectorial objective function $\mathbf{f}_{i}^{A}$, where:

$$
\mathbf{f}_{i}^{A}=\left[f_{1, i}^{A}, f_{2, i}^{A}, \ldots, f_{k, i}^{A}\right]^{T}
$$

The dominance of an element $i$ of strategy $A$ with respect to strategy $B$ is the cardinality of the set of dominated components.

$$
I_{i}\left(m_{A}\right)=\left|\left\{j \mid \mathbf{f}_{i}^{A} \prec \mathbf{f}_{j}^{B}\right\}\right|
$$

Hence strategy $A$ dominates strategy $B$ if the sum of the elements of $A$ that are dominated by $B$ is less than the sum of the elements of $B$ that are dominated by $A$.

$$
\begin{aligned}
& d_{i}=\left\{\begin{array}{l}
1 \text { if } I_{i}>0 \\
0 \text { if } I_{i}=0
\end{array}\right. \\
& m_{A} \succ m_{B} \Rightarrow \frac{1}{N_{A}} \sum_{i=1}^{N_{A}} d_{i}\left(m_{A}\right)<\frac{1}{N_{B}} \sum_{j=1}^{N_{B}} d_{j}\left(m_{B}\right)
\end{aligned}
$$

Where $N_{A}$ is the total number of the solutions in the Pareto front of method (or strategy) $m_{A}$ and $N_{B}$ is the total number of solutions in the Pareto front of method (or strategy) $m_{B}$. The dominance of the different strategies is shown in Tables $2-4$.

TABLE 2. Nyx Comparison Table (a=1.93 A.U.).

\begin{tabular}{c|ccccc}
\hline & Nuclear Blast & Kinetic Impactor & Solar Mirror & Mass Driver & Low-Thrust \\
\hline Nuclear Blast & 0 & 0 & 79.86 & 0 & 0 \\
Kinetic Impactor & 55.63 & 0 & 100 & 100 & 98.33 \\
Solar Mirror & 50.15 & 0 & 0 & 2.04 & 0 \\
Mass Driver & 89.93 & 0 & 93.63 & 0 & 0 \\
Low-Thrust & 89.66 & 0 & 100 & 100 & 0 \\
\hline
\end{tabular}

TABLE 3. Castalia Comparison table ( $\mathrm{a}=1.06$ A.U.).

\begin{tabular}{c|ccccc}
\hline & Nuclear Blast & Kinetic Impactor & Solar Mirror & Mass Driver & Low-Thrust \\
\hline Nuclear Blast & 0 & 0 & 93.06 & 0 & 0 \\
Kinetic Impactor & 98.64 & 0 & 100 & 100 & 0 \\
Solar Mirror & 2.03 & 0 & 0 & 0 & 0 \\
Mass Driver & 95.92 & 0 & 100 & 0 & 0 \\
Low-Thrust & 91.81 & 24.96 & 100 & 100 & 0 \\
\hline
\end{tabular}

TABLE 4. Apophis Comparison Table (a=0.92 A.U.).

\begin{tabular}{c|ccccc}
\hline & Nuclear Blast & Kinetic Impactor & Solar Mirror & Mass Driver & Low-Thrust \\
\hline Nuclear Blast & 0 & 0 & 36.99 & 0 & 0 \\
Kinetic Impactor & 99.32 & 0 & 99.94 & 99.45 & 80.89 \\
Solar Mirror & 0.73 & 0 & 0 & 0 & 0 \\
Mass Driver & 96.52 & 0 & 99.95 & 0 & 0 \\
Low-Thrust & 99.95 & 0 & 99.95 & 100 & 0 \\
\hline
\end{tabular}

The results in Tables 2 to 4 are the percentage of dominance of one deviation strategy over another one. The value in each cell of the tables represents the dominance of the strategy in the corresponding column over the one in the corresponding row. So the value in position $\left[\mathrm{row}_{B}, \mathrm{colum}_{A}\right]$ represents the dominance of strategy $A$ on strategy $B$; and vice versa the dominance of strategy $B$ on strategy $A$ is in cell $\left[\right.$ row $_{A}$, column $\left._{B}\right]$. For example in Table 4 
(Apophis), only $0.73 \%$ of the solutions of the nuclear strategy dominate the ones of the solar method, while the solar strategy dominates the nuclear by $36.99 \%$. The grey background in the Tables indicates when one method clearly dominates over the other methods presented.

In most of the cases the sum of the two percentages is not 100; this is due to two reasons: Firstly $N_{A}$ is not necessarily equal to $N_{B}$, and secondly, because in certain areas none of the two methods is dominating over the other (e.g. Solar and Nuclear dominance for Apophis). These figures have an inherent margin of error since the Pareto sets for each strategy are only composed of a finite and discrete number of elements. More accurate numbers can be computed by increasing the number of solutions, uniformly distributed, in each Pareto set.

\section{Influence of the Technology Readiness Level}

As an additional criterion we considered the technology readiness level (TRL) of each method as a measure of the expected reliability. We define a TRL factor or TRL $f$ by mapping a scale from 1 to 8 (see Table 5) onto the interval [0 1], where a TRLf equal to 1 means full operational capability.

TABLE 5. Technology Readiness Levels.

\begin{tabular}{ll}
\hline $\mathbf{1}$ & No development performed \\
$\mathbf{2}$ & Conceptual design formulated \\
$\mathbf{4}$ & Conceptual design tested analytically or experimentally \\
$\mathbf{5}$ & Critical function/characteristic demonstrated \\
$\mathbf{6}$ & Component or Breadboard tested in relevant environment \\
$\mathbf{7}$ & Prototype/engineering model tested in relevant environment \\
$\mathbf{8}$ & Engineering model/similar equipment tested in space \\
\hline
\end{tabular}

For every deviation methodology a certain $T R L$ has been determined, taking into account past missions and experiments. Moreover, even though the strategies were modeled with a very conservative approach, an additional margin on the initial spacecraft mass has been added in order to take into account the corrective maneuvers required during both the transfer leg and the deflecting arc. These margins differ among the various deviation methods due to the different levels of development of the required technology. The TRL $f$ and the mass margins are presented in Table 6.

TABLE 6. $T R L f$ and margins on the wet mass into orbit for the different deviation strategies.

\begin{tabular}{lll} 
Strategies & TRLf & $\boldsymbol{m}_{\boldsymbol{0}}$ margin \\
Solar Collector & 0.375 & $25 \%$ \\
Electric Propulsion & 0.5 & $25 \%$ \\
Mass Driver & 0.375 & $25 \%$ \\
Nuclear Impactor & 0.5 & $10 \%$ \\
Kinetic Impactor & 1 & $5 \%$ \\
\hline
\end{tabular}

Despite the fact that no mission to test this technology is flying yet, the technology for the Kinetic Impactor has been considered to be fully developed, TRL 8. Examples of this technology are plentiful (e.g. Deep Impact [3]) and the kinetic deflection mission Don Quijote scheduled for a 2011 launch [2]. Nuclear and Low-Thrust technologies have been considered to be at an intermediate stage where the critical function and characteristics have been demonstrated. The main reason is that the environment in which the technology would be used is completely different from that for which it was designed and tested. Finally, Solar Collector and Mass Drivers are assumed to be at TRL 3, since basic experiments with this technology have already been performed, examples are the deployment of inflatable structures [34] or the autonomous drilling of planetary surfaces, etc.

A fixed impact date was taken for all the strategies applied to a given asteroid in order to analyze the effects of each strategy for the same MOID. As a consequence, the effect of the technology readiness level is to postpone the departure time by a percentage of the warning time proportional to the TRLf of each strategy. This was implemented by setting a different lower boundary for the departure time for each analyzed strategy, while the upper boundary has been kept fixed. The outcome of this approach is a shrinking of the $t_{w}$ in the Pareto Front, depending on the $T R L f$ of each strategy. 
TABLE 7. Apophis $T R L$ Comparison Table ( $\mathrm{a}=0.92$ A.U.).

\begin{tabular}{l|ccccc}
\hline & Nuclear Blast & Kinetic Impactor & Solar mirror & Mass Driver & Low-Thrust \\
\hline Nuclear Blast & 0 & 0 & 78.78 & 0 & 0 \\
Kinetic Impactor & 100 & 0 & 99.56 & 97.23 & 39.68 \\
Solar Mirror & 14.27 & 0.13 & 0 & 0.13 & 0.13 \\
Mass Driver & 100 & 0.08 & 99.95 & 0 & 0.05 \\
Low-Thrust & 100 & 4.6 & 99.97 & 99.48 & 0 \\
\hline
\end{tabular}

Table 7 shows the dominance of the analyzed strategies for the case of Apophis after applying the TRL factor. By comparing this Table with Table 4, few preliminary considerations can be drawn. The first consideration is that when the Technology Readiness Level is considered, the Kinetic Impactor becomes competitive since its Pareto front encloses parts of the criteria domain that the other strategies are not able to cover; this is a direct consequence of the $t_{w}$ shrinking. On the other hand, even after the technology readiness filtering, the Solar Mirror strategy remains particularly competitive. For example, for the Apophis case (Table 7), the Solar Mirror almost completely dominates the Nuclear Blast option.

The Mass Driver method cannot be completely discarded, because it has a satisfactory behavior. This is even truer when this strategy is applied to small asteroids with large semi-major axes. Finally the technology readiness analysis reveals the impracticability of the Low-Thrust attached device. In fact, the achieved results are comparable with the Kinetic Impactor, despite the more complex technology of the Low-Thrust devices.

\section{FINAL REMARKS}

In this work, different strategies to deviate dangerous NEOs have been modeled and compared. A set of analytical formulas have been proposed to compute the variation of the MOID due to a given deviation strategy. The variation has been expressed as a function of the warning time and the wet mass of the spacecraft at the Earth.

The comparison has been performed on three classes of asteroids, by means of a multi-criteria optimization. The sets of Pareto optimal solutions for each strategy have been compared by defining the dominance of one Pareto set over another. Moreover a technology readiness factor has been introduced in order to estimate the actual required warning time.

This preliminary comparison shows how the solar concentrators and nuclear blast are generally dominant. The Solar Mirror method remains a very competitive technology even after TRL considerations. On the other hand Kinetic Impactor may be still a very reasonable method for small asteroids, since it is able to achieve deviations of several Earth radii with an affordable mass. The definition of the TRL factor is of course arbitrary and is subject to the actual development of each specific piece of technology that composes a given strategy. However for the Kinetic Impactor no technology development is required at present and it is therefore the only strategy already available. The percentage of dominance for the other methods, instead, is expected to change according to the future investment in one technology or the other.

A more accurate comparison would require an improvement of all the models as well as taking into account a possible fragmentation of the asteroid due to an overly aggressive action. Moreover a more complete reproduction of the actual Pareto sets would provide more accurate figures for the percentage of dominance.

Finally the analysis presented in this paper was limited to a small group of asteroid. A more exhaustive analysis comprising a larger group of asteroids is currently being performed and will give an indication on the most effective strategy for a given class of asteroids.

\section{REFERENCES}

1. Naja G., "The Second Report by ESA's Long-Term Space Policy Committee (LSPC)", Directorate of Strategy and Technical Assessment, ESA, Paris, May 1999.

2. Don Quijote Homepage: http://www.esa.int/gsp/completed/neo/donquijote.html.

3. Deep Impact Homepage: http://deepimpact.jpl.nasa.gov.

4. NEAR mission Homepage: http://near.jhuapl.edu.

5. Rosetta mission Homepage: http://www.esa.int/esaMI/Rosetta.

6. Scheeres D. J., Schweickart R. L., "The Mechanics of Moving Asteroids", 2004 Planetary Defense Conference: Protecting Earth from Asteroids, 23-26 February 2004, Orange Country, California.

7. Bourdoux A., Izzo D., "Characterization and hazard mitigation of resonant returning Near Earth Objects", Final Stage Report, ACT internal report: ACT-RPT-4100-AB-CHMRRNEO05. 
8. Valsecchi G. B., Milani A., Gronchi G. F., Chesley S. R., "Resonant returns to close approaches: Analytical theory", Astronomy and Astrophysics, 2003, pp. 1179-1196.

9. Carusi A., Valsecchi G. B., D’abramo G., Bottini A., "Deflecting NEOs in Route of Collision with the Earth", Icarus, Vol. 159, 2002, pp. 417-422.

10. Park S.-Y., Ross I. M., "Two-Body Optimization for Deflecting Earth-Crossing Asteroids”, Journal of Guidance, Control and Dynamics, Vol. 22, No. 3, 1999, pp. 995-1002.

11. Ross I. M., Park S.-Y., Porter S. D. V., "Gravitational Effects of Earth in Optimizing $\Delta v$ for Deflecting Earth-Crossing Asteroids", Journal of Spacecraft and Rockets, Vol. 38, No. 5, September-October 2001, pp. 759-764.

12. Conway B. A., "Near-Optimal Defection of Earth-Approaching Asteroids", Journal of Guidance, Control and Dynamics, Vol. 24, No. 5, 2001, pp. 1035-1037.

13. Tedeschi W. J., Remo J. L., Schulze J. F., Young R. P., "Experimental Hypervelocity Impact Effects on Simulated Planetesimal Materials", International Journal of Impact Engineering, Vol. 17, Elsevier Science Ltd, 1995, pp. 837-848.

14. McInnes C. R., "Deflection of near-Earth asteroids by kinetic energy impacts from retrograde orbits", Planetary and Space Science, Vol. 52, Elsevier, 2004, pp. 587-590.

15. Ivashkin V. v., Smirnov V. V., "An analysis of some methods of asteroid hazard mitigation for the Earth", Planetary Space Science, Vol. 43, No. 6, Elsevier Science Ltd, 1995, pp. 821-825.

16. Spitale J. N., “Asteroid Hazard Mitigation Using the Yarkovsky Effect”, Science, Vol. 295, 5 April 2002, pp. 77.

17. Melosh H. J., Nemchinov I. V., Zetzer Y. I., "Non-nuclear strategies for deflecting comets and asteroids" in Gehrels T. (editor), Hazard Due to Comets and Asteroids, University of Arizona Press, Tucson, 1994, pp. 1111- 1132.

18. Hall C. D., Ross I. M., "Dynamic control problems in the deflection of near-Earth objects", AAS 97-640, 1997.

19. Canavan G. H., Solem J. C., Rather J. D. G. (editors), Proceeding of the Near-Earth-Object Interception Workshop, Los Alamos National Laboratory, 1992.

20. Schaub H., Junkins J. L., Analytical mechanics of space systems, AIAA Education Series, AIAA, 2003, pp. $592-623$.

21. Battin R. H., An Introduction to the Mathematics and Methods of Astrodynamics, Revised Edition, AIAA Education Series, AIAA, 1999.

22. Maddock C., Sanchez Cuartielles J. P., Vasile M., Radice G., Comparison of Single and Multi-Spacecraft Configurations for NEA Deflection by Solar Sublimation, Reviewing process by the AIP. 2006.

23. Kahle R., Kührt E., Hahn G., Knollenberg J., "Physical Limits of Solar Collectors in Deflecting Earth-threatening Asteroids", Aerospace Science and Technology, Vol. 10, 2006, pp. 256-263.

24. Wertz J. R., Larson W. J., Space Mission Analysis and Design, Third ed., Microcosm Press 2003.

25. Olds J., Charania A., Graham M., Wallace J., "The League of Extraordinary Machines: A rapid and Scalable Approach to Planetary defense Against Asteroid Impactors", 2004.

26. Ahrens T. J., Harris A. W., "Deflection and Fragmentation of Near-Earth Asteroids", Nature, Vol. 360, 1992, pp. 429-433.

27. Remo J. L., "Energy Requirements and Payload Masses for Near-Earth Objects Hazard Mitigation", Acta Astronautica, Vol. 47, No. 1, 2000, pp. 35-50.

28. Smith P. L., Barrera M. J., Campbell E. T., Fedman K. A., Peterson G. E., Smith G. N., "Deflecting a Near Earth Object with Today's Space Technology," 2004 Planetary Defense Conference: Protecting Earth from Asteroids, 2004.

29. Kleinman L. A. (Ed.), Project ICARUS. MIT Report 13, MIT Press, Cambridge (1968). Called "ICARUS report" in the text.

30. Tedeschi W. J., Remo J. L., Schulze J. F., Young R. P., International Journal of Impact Engineering, Vol. 17, 837 (1995).

31. Wang J., Davis A. M., Clayton R. N., Hashimoto A., Geochimica Et Cosmochimica Acta, Vol. 63(6), 953 (1999).

32. De Pascale P., Vasile M., "Preliminary Design of Low-thrust Multiple Gravity-Assist Trajectories". Journal Of Spacecraft and Rockets, 2006, in press.

33. Vasile M., "A Hybrid Multi-Agent Collaborative Search Applied to the Solution of Space Mission Design Problems", Proceeding of Global Optimisation, 2005, pp. 1-11.

34. Thomas M., "Inflatable Space Structures", IEEE Potentials Magazine, 1992, pp. 29-32.

35. Conway B. A., "Optimal Low-Thrust Interception of Earth-Crossing Asteroids", Journal of Guidance, Control and Dynamics, Vol. 20, No. 5, 1997, pp. 995-1002.

36. Kahle R., Hahn G., Kührt E., “Optimal deflection of NEOs en route of collision with the Earth”, Icarus, Vol. 182, 2006, pp. 482-488. 